\title{
Hormonal and metabolic response to hypoglycaemia in small for gestational age infants
}

\author{
J M Hawdon, A Weddell, A Aynsley-Green, M P Ward Platt
}

\begin{abstract}
Little is known of the ability of hypoglycaemic infants who are small for gestational age (SGA) to mount the coordinated hormonal and metabolic counterregulatory response that is seen in healthy older subjects during glycopenia. This response was studied in 22 SGA infants (birth weight <10th centile) by measuring the blood concentrations of glucose, intermediary metabolites, and glucoregulatory hormones.

Plasma non-esterified fatty acid and blood ketone body concentrations were low, even when blood glucose concentrations were low. Plasma insulin and glucagon varied widely $(<1 \cdot 0-53 \cdot 1 \mathrm{mU} / 1$ and $16 \cdot 6-87 \cdot 1 \mathrm{pmol} / \mathrm{l}$, respectively). Concentrations of noradrenaline and glucagon were raised, but cortisol and adrenaline were lower than those found in hypoglycaemic adults. There was no relationship between the concentration of any hormone and blood glucose concentration.

We postulate that hypoglycaemia and the failure to mobilise alternative fuels in some SGA infants is secondary both to a poorly coordinated counterregulatory hormone response and to a peripheral insensitivity to the actions of the hormones. Those infants, who fail to mount a counterregulatory response, should be identified by accurate and reliable blood glucose monitoring, and an adequate exogenous supply of energy, either enteral or parenteral, should be ensured.
\end{abstract}

(Arch Dis Child 1993;68:269-273)

Department of Child Health, University of Newcastle upon Tyne J M Hawdon

A Aynsley-Green

M P Ward Platt

Department of Clinical Biochemistry,

Newcastle General

Hospital

A Weddell

Correspondence to:

Dr J M Hawdon, Institute of Child Health, Royal

Liverpool Children's

Hospital Alder Hey, Eaton

Road, Liverpool L12 2AP.

Accepted 25 August 1992 regulatory response to hypoglycaemia is deficient. ${ }^{4}$ The respective metabolic responses to hypoglycaemia in appropriate weight for gestational age (AGA) and SGA infants have been compared ${ }^{5} 6$ and it has been shown that AGA infants, like adults, mount a ketogenic response, but that this is deficient in many SGA infants. It is not known whether this is, in turn, the result of a deficient hormonal counterregulatory response. To date, the simultaneous measurement of glucoregulatory hormones, in the context of the metabolic response to low blood glucose concentrations, has not been reported in a homogenous group of babies. The present study examined the profiles of metabolic fuels and counterregulatory hormones in SGA infants, in order to identify possible deficiencies in the hormonal and metabolic response to hypoglycaemia.

\section{Subject and methods}

The subjects were 22 SGA neonates (birth weight $<10$ th centile) of mixed gestation, cared for in two neonatal units, who were thought to be hypoglycaemic on the day of study (blood glucose concentration $<2.6$ $\mathrm{mmol} / 1$ on at least two occasions), and for whom a clinical decision had been made to treat with intravenous glucose infusion. Blood glucose monitoring was by BM stick measurement (BM Diagnostics) on one neonatal unit (12 babies), and by immediate laboratory measurement, using a Yellow Springs analyser, on the other unit (10 babies). No infant had a coexisting clinical complication.

Blood samples were collected from each baby before treatment with intravenous glucose or before the increase in glucose infusion rate if the baby was already receiving intravenous glucose. For ethical reasons, treatment was not delayed by the baby's inclusion in the study. A sample of $1.5 \mathrm{ml}$ free flowing blood was taken for subsequent measurement of blood concentrations of glucose and intermediary metabolites and plasma glucoregulatory hormone. Blood for plasma glucagon assay was collected into a tube containing aprotinin. Sample tubes were stored on ice during collection, and plasma for hormone assay was immediately separated and stored at $-80^{\circ} \mathrm{C}$.

Concentrations of blood glucose, lactate, pyruvate, alanine, glycerol, ketone bodies, and plasma non-esterified fatty acids were assayed as described previously. ${ }^{5}$ Plasma insulin, cortisol, and glucagon concentrations were determined by radioimmunoassay, ${ }^{7-9}$ and a radioenzymatic method was used for the assay of plasma adrenaline and noradrenaline. ${ }^{10}$ 
Data were analysed using the SPSS-X package. Blood concentrations of lactate, pyruvate, alanine, and glycerol were summed to give total gluconeogenic substrate concentration. For this cohort of infants the concentrations of metabolites and hormones were found not to have a normal distribution, and some infants had circulating ketone body, glucose, and insulin concentrations that were at the lower level of assay sensitivity. Therefore, non-parametric tests were used, with Spearman rank correlation to assess relationships between variables.

For each infant, the mid-arm circumference:head circumference ratio (MAC:HC) was calculated as a measure of the degree of asymmetry of growth retardation. ${ }^{11}$ Birthweight and MAC:HC SD scores were calculated, that is the number of SDs these measures lay from the 50th centile.

Ethical approval for the study was granted by the ethics committee of Newcastle Health Authority, and parents gave informed consent for inclusion of their baby in the study.

\section{Results}

Details of the infants are given in the table. All infants were at least 9 hours old and all had received enteral milk feeds; samples were taken at least one hour after the last enteral feed. Eight infants were receiving intravenous glucose at the time samples were taken. None of the infants had clinical symptoms associated with hypoglycaemia, and they had no other systemic disorder.

Four babies had true blood glucose concentrations $>3.0 \mathrm{mmol} / \mathrm{l}$ at the time of commencement of the study. For all four of these babies, diagnosis of hypoglycaemia was originally made by BM stick estimation of blood glucose concentrations. Eight babies had true blood glucose concentrations $2 \cdot 6-3 \cdot 0$ $\mathrm{mmol} / \mathrm{l}$, and 10 babies had true blood glucose $<2.6 \mathrm{mmol} / \mathrm{l}$ when the study commenced.

Figure 1 demonstrates the interrelationships between fuel concentrations. There were no significant relationships between blood glucose and total gluconeogenic substrate concentrations. Plasma non-esterified fatty acid and blood ketone body concentrations were low. The negative relationships between blood glucose concentrations and the respective ketone body and non-esterified fatty acid concentrations, seen in AGA infants, ${ }^{5}$ were absent. There was no significant relationship between fatty acid and ketone body concentrations for the babies of the present study. The metabolite concentrations were not related to feed parameters such as feed volume

\begin{tabular}{lll}
\multicolumn{3}{l}{ Clinical details of infants studied } \\
\hline & Median & Range \\
\hline Gestation (weeks) & 37 & $30-40$ \\
Birth weight (g) & 2050 & $1150-2700$ \\
Birthweight SD score & $-2 \cdot 58$ & $-3 \cdot 72$ to $-1 \cdot 69$ \\
MAC:HC SD & $-1 \cdot 02$ & $-4 \cdot 96$ to $1 \cdot 18$ \\
Age (hours) & 28 & $9-340$ \\
IV glucose (mg/kg/min) & 0 & $0-8 \cdot 6$ \\
Milk (mg/kg/day) & 100 & $41-167$ \\
Time since last feed (min) & 120 & $60-240$ \\
Energy intake (kJ/kg/day) & 397 & $138-589$ \\
\hline
\end{tabular}

and the time since the last feed.

The concentrations of glucoregulatory hormones in relationship to blood glucose concentration are shown in fig 2 . It is evident that many infants had high plasma insulin concentrations. There was no relationship between insulin concentration and postnatal age. Concentrations of counterregulatory hormones varied widely, and no relationship existed between the concentration of any glucoregulatory hormone and blood glucose. Neither were non-esterified fatty acid and ketone body concentrations related to that of any hormone. As for metabolite concentrations, hormone concentrations were not related to feed parameters.

\section{Discussion}

In a previous longitudinal study of a separate group of SGA infants, we have identified reduced concentrations of alternative fuels, even when blood glucose was low. ${ }^{6}$ The present study supports these metabolic
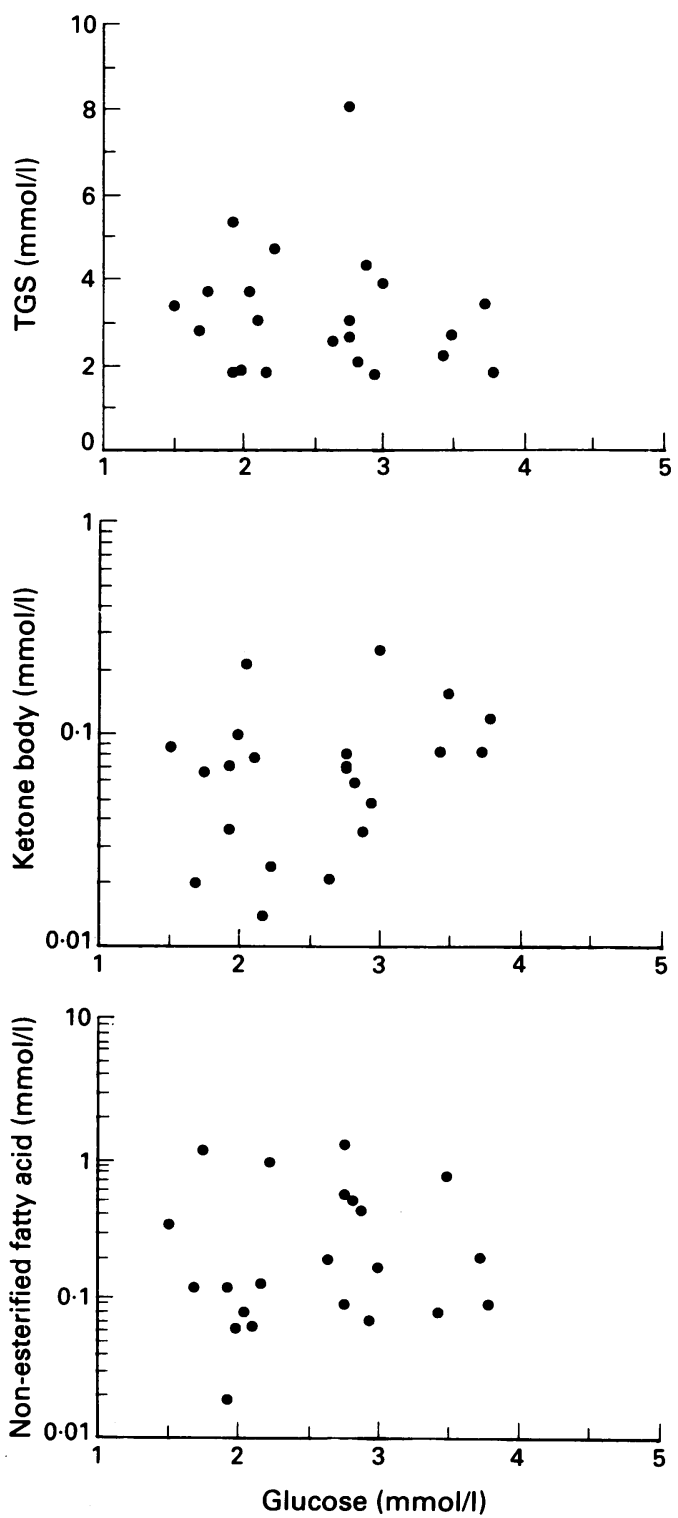

Figure 1 Relationship of blood total gluconeogenic substrate (TGS), ketone body, and non-esterified fatty acid concentrations with blood glucose concentration. 

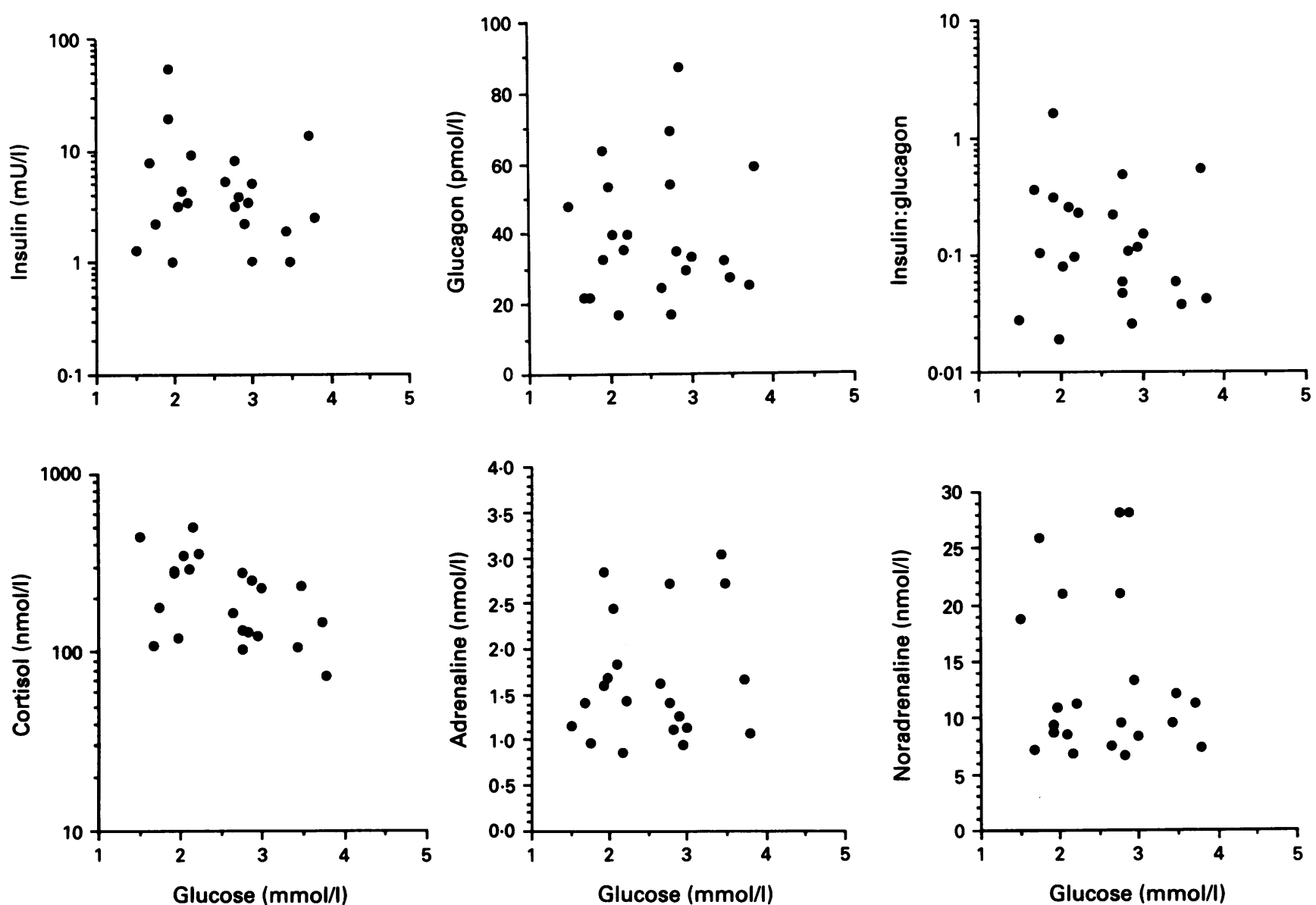

Figure 2 Relationship of plasma glucoregulatory hormone concentrations with blood glucose concentration.

findings, namely that SGA infants have reduced lipolytic and ketogenic capacity. As in the previous study it is apparent that the reduced ketone body concentrations may arise both from deficient lipolysis, as evident by low non-esterified fatty acid concentrations, and deficient ketogenesis, as evident by the absence of a relationship between nonesterified fatty acid and ketone body concentrations. These metabolic interrelationships are discussed in more detail elsewhere. ${ }^{6}$

In addition to the data previously described, the present study documents the full hormonal and metabolic profile, and postulates that hormonal mechanisms may contribute to hypoglycaemia and the failure of counterregulatory ketogenesis.

Many studies of glucoregulatory hormone concentrations in SGA infants have found few endocrine differences between SGA and AGA infants when normoglycaemic. ${ }^{12-15}$ However, the hormonal response of SGA infants to hypoglycaemia is of greater clinical importance and has been described in more detail in the present study. This will be discussed in two parts: first, the role of hyperinsulinism and second, the counterregulatory hormonal response.

Adult studies have demonstrated that insulin secretion is suppressed at blood glucose concentrations $<3.0 \mathrm{mmol} /{ }^{1}$ However, neonatal studies have suggested that hyperinsulinaemic hypoglycaemia may occur in SGA infants. ${ }^{16} 17 \mathrm{~A}$ recent review of the literature has suggested that possible under- lying mechanisms for hyperinsulinaemia are $\beta$ cell hyperplasia, increased sensitivity of $\beta$ cells to glucose, or increased insulin release in response to stress, as for shocked adult patients. ${ }^{18}$ These previous studies mainly described infants less than 6 hours old, some of whose mothers received intravenous glucose during labour, and for whom concentrations of other glucoregulatory hormones were not documented. We have previously demonstrated that this is a period during which many metabolic changes occur, even in AGA infants, ${ }^{5}$ and it is well known that administration of glucose to mothers in labour affects early neonatal blood glucose concentrations. ${ }^{19}$ In addition, the insulin/glucose relationship for healthy term neonates, of the same postnatal age, has not yet been documented. Therefore, conclusions drawn from the previous studies of such young infants may not be valid.

The present study excluded infants less than 9 hours old, and no mother received intravenous glucose in labour. We have demonstrated that many SGA infants had high insulin concentrations, even when hypoglycaemic. With the exception of one infant, all subjects with blood glucose $<3 \mathrm{mmol} / \mathrm{l}$ had measurable insulin concentrations (fig 2). This suggests that hyperinsulinism, persisting after the immediate postnatal period, may have contributed to hypoglycaemia.

The subjects of the present study had raised glucagon concentrations, which were similar to those found in hypoglycaemic 
adults. ${ }^{1-3}$ Animal studies have demonstrated that low insulin:glucagon ratios are essential for induction of phosphoenolpyruvate carboxykinase, the major gluconeogenic enzyme, with a reduction of activity of $60 \%$ if the ratio is greater than $2 .{ }^{20} \mathrm{In}$ addition, adult human studies have demonstrated that the ratio is reduced to less than 2 in the fasted state. ${ }^{21}$ All of the infants in the present study had ratios below this level. If circulating plasma hormone concentrations reflect the rate of pancreatic secretion, this would imply that the effects of high circulating insulin concentrations should have been balanced by glucagon activity. The presence of hypoglycaemia in the context of appropriately raised glucagon concentrations suggests that the peripheral response to glucagon was impaired. This study was not able to investigate the relationship between circulating hormone concentrations and pancreatic secretory activity nor the role of hormone receptor activity in the failure of metabolic adaptation.

Insulin is the major hormone controlling lipolysis, and glucagon promotes ketogenesis by the liver. ${ }^{22}$ Therefore, high insulin concentrations will inhibit lipolysis, regardless of glucagon concentrations, and thus reduce the availability of substrate for ketogenesis. It is possible that this was the mechanism for hypoketonaemia in the present study, but ketone body turnover studies would be required to confirm this.

There is evidence from animal studies that neonates do not have a fully functional sympathoadrenal response to hypoglycaemia, and it has been suggested that this is secondary to an immaturity of neural pathways. ${ }^{23} 24$ This was not supported by a study of human neonates, which demonstrated a marked noradrenergic response to hypoglycaemia. ${ }^{25}$ However, in this latter study, hypoglycaemia was induced by abruptly discontinuing high rate glucose infusion and the only SGA infant studied had systemic complications, so the findings may not be applicable to spontaneously occurring hypoglycaemia in otherwise well SGA infants. Increased urinary excretion of catecholamines has been demonstrated for SGA infants during hypoglycaemia but, unfortunately, this particular study did not record plasma catecholamine concentrations. ${ }^{26}$

Adrenaline concentrations varied little among the infants in the present study, were similar to those found in moderately hypoglycaemic adults (blood glucose $>2.0 \mathrm{mmol} / \mathrm{l}$ ), but were lower than those found in severely hypoglycaemic adults (blood glucose $\leq 2.0$ $\mathrm{mmol} / \mathrm{l}) .^{2}{ }^{3}$ On the other hand, there was a wide variation in plasma concentrations of noradrenaline, and concentrations were higher than those recorded for even severely hypoglycaemic adults. However, there was no relationship between blood glucose and plasma catecholamine concentration, which suggests that the catecholamine response was not to hypoglycaemia alone, but may have represented a general stress response in this group of infants.
The plasma cortisol concentrations, described in the present study, were not high either in terms of the neonatal stress response reported by other authors or the adult counter-regulatory response to hypoglycaemia. $^{2} \quad 3 \quad 27 \quad 28$ Neither were cortisol concentrations related to blood glucose concentrations. This suggests that SGA infants may have diminished adrenocorticol secretory ability.

Non-esterified fatty acid concentrations were low, even in the presence of raised noradrenaline concentrations, and were not related to noradrenaline concentrations, which suggests that lipolysis was inhibited, perhaps by high circulating insulin concentrations.

In summary, we demonstrated the presence of relative hyperinsulinaemia and an incomplete counterregulatory response to hypoglycaemia in SGA infants who were otherwise well. We postulate that hyperinsulinaemia alone was not responsible for hypoglycaemia, as raised plasma glucagon concentrations maintained a low insulin:glucagon ratio. However, the absence of a coordinated hormonal counterregulatory response may have contributed to the persistence of hypoglycaemia. The availability of alternative fuels to glucose is of importance for cerebral metabolism in the neonatal period, and we suggest that the abnormalities of hormonal response, demonstrated in the present study, contributed to the failure to provide alternative fuels in these SGA infants.

We were unable to assess the role of end organ response to the action of these hormones. Glucose, fatty acid, and ketone body turnover studies would give further information regarding the effects of glucoregulatory hormones on hepatic glucose production in SGA infants. These studies could investigate the response both to endogenous hormone secretion, and to the exogenous administration of hormones. Interpretation of single data points, as in the present study, assumes that circulating hormones and metabolite concentrations reflect turnover rates. In such clinical studies it is difficult to test this hypothesis as steady state is required to assess these relationships. For example, in the present study, the clinical and ethical requirement to treat hypoglycaemia without delay precluded repeated sampling from each baby under stable conditions before correction of hypoglycaemia.

The subjects of the present study were chosen because there was clinical concern regarding blood glucose homoeostasis, this concern was justified by our findings. While the aim of this study was not to investigate the reliability of BM stick measurements for the diagnosis of hypoglycaernia, note should be made that, of 12 babies diagnosed as hypoglycaemic by BM stick measurement, four were subsequently found to have blood glucose concentrations well within the normal neonatal range. ${ }^{5}$ This demonstrates the importance of accurate and reliable diagnostic 
methods for blood glucose monitoring in the neonatal period.

Though not all SGA infants experience metabolic difficulties, those who are unable to mount an adequate counterregulatory response to hypoglycaemia are unlikely to spontaneously revert to normoglycaemia or to provide, for themselves, alternative fuels. Therefore, this latter group of infants should be identified by accurate blood glucose monitoring, and an adequate exogenous supply of enteral and parental energy must be ensured.

Dr Hawdon was supported by the Scientific and Research Committee of Newcastle Health Authority and by a Foundation for the Study of Infant Deaths training fellowship. We thank the parents who gave consent for the inclusion of their babies in the study. We acknowledge the assistance of the their babies in the study. We acknowledge the assistance of the following: Ms A McGann, Department of Child Health, Newcastle University, with metabolite assays, Dr M Ghatei
and Professor S Bloom, Hammersmith Hospital, London, with and Professor S Bloom, Hammersmith Hospital, London, with glucagon assays, Dr G Dale, Department of Clinical Biochemistry, Newcastle General Hospital, with cortisol assays, Mr M Ashby and Professor M Brown, Department of Clinical Pharmacology, Addenbrooke's Hospital, Cambridge, with catecholamine assays, and Ms L Ashworth and Professor Medicine, Newcastle University, with insulin assays.

1 Gerich JE. Glucose counterregulation and its impact on diabetes mellitus. Diabetes 1988;37:1606-17.

2 Gerich J, Davis J, Lorenzi M, et al. Hormonal mechanisms of recovery from insulin-induced hypoglycaemia in man. Am $\mathcal{F}$ Physiol 1979;236:E380-5.

3 Rizza R, Cryer P, Gerich J. Role of glucagon, catecholamines and growth hormone in human glucose councholamines and growth hormone in human glucose counand $\beta$ adrenergic blockade and plasma glucose recovery and $\beta$ adrenergic blockade and plasma glucose recovery and glucose flux rates following insulin-

4 Hetenyi G, Cowan JS. Glucoregulation in the newborn. Can $\mathcal{F}$ Physiol Pharmacol 1980;58:879-88.

5 Hawdon JM, Ward Platt MP, Aynsley-Green A. Patterns of metabolic adaptation for preterm and term infants in the first neonatal week. Arch Dis Child 1992;67:357-65.

6 Hawdon JM, Ward Platt MP. Metabolic adaptation in small for gestational age infants. Arch Dis Child 1993; 68:262-8.

7 Soeldner JS, Slone D. Critical variables in the RIA of serum insulin using the double antibody system. Diabetes 1965;14:771-9.

8 Lantto O, Lindback B, Aakvaag A, Damjaer-Nielsen M, Pomoell V. Assay of cortisol with a radioimmunoassay method calibrated by isotopic dilution mass spectrometry. A nordic collaborative study. Scand f Clin Lab Invest 1983; 43: 433-7.
9 Christofides ND. Pancreatic glucagon. In: Bloom SR, Long $\mathrm{R}$, eds. Radioimmunoassay of gut regulatory peptides. London: WB Saunders, 1982.

10 Brown MJ, Jenner DA. Novel double-isotope technique for enzymatic assay of catecholamines, permitting high precision sensitivity and plasma sample capacity. Clin Sci sion, sensitivity

11 Sasanow SR, Georgieff MK, Pereira GR. Mid-arm circumference and mid-arm circumference/head circumference ratios: standard curves for anthropometric assessmen
neonatal nutritional status. $\mathcal{f}$ Pediatr 1986;109:311-5.

12 Cowett RM, Susa JB, Oh W, Schwartz R. Glucose kinetics in glucose infused small for gestational age babies. Pediat Res 1984;18:74-9.

13 Salle BL, Ruiton-Ügliengo A. Effects of oral glucose and protein load on plasma glucagon and insulin concentrations in small for gestational age infants. Pediatr Res 1977; 11:108-12.

14 Rokicki W, Forest MG, Loras B, Bonnett H, Bertrand J. Free cortisol of human plasma in the first three months of life. Biol Neonate 1990;57:21-9.

15 Doerr HG, Vermold HT, Bidlingmaier F, Sippell WG. Adreno-cortical steroids in small for gestational age term infants during the early neonatal period. Pediatr Res 1989; 25:115-8.

16 Le Dune MA. Intravenous glucose tolerance and plasma insulin studies in small for dates infants. Arch Dis Child 1972;47:111-4.

17 Collins JE, Leonard JV, Terle D, et al. Hyperinsulinaemic hypoglycaemia in small for dates babies. Arch Dis Child 1990;65:1118-20.

18 Bhowmick SK, Lewandowski C. Prolonged hyperinsulinism and hypoglyceamia in an asphyxiated small for gestation infant. Case management and literature review. Clin Pediatr (Phila) 1989;28:575-8.

19 Lucas A, Adrian TE, Aynsley-Green A, Bloom SR. Iatrogenic hyperinsulinism at birth. Lancet 1980;i:144-5.

20 Girard J. Gluconeogenesis in the late fetal and early neonatal life. Biol Neonate 1986;50:237-58.

21 Unger RH. Glucagon and the insulin:glucagon ratio in diabetes and other metabolic illness. Diabetes 1971;20 834-8.

22 McGarry JD, Foster DW. Ketogenesis. In: Rifkin H, Porte $\mathrm{D}$, eds. Ellenberg and Rifkin's diabetes mellitus. Theory and practice. New York: Elsevier, 1990.

23 Silver M, Fowden AC, Knox J, Ousey JC, Franco R, Rossdale PD. Sympathoadrenal and other responses to hypoglycaemia in the young foal. $\mathcal{F}$ Reprod Fertil 1987; 35 (suppl):607-14.

24 Lau C, Bartolome JV, Bartolome MB, Slotkin TA. Centra and synmpathoadrenal responses to insulin in adult and neonatal rats. Brain Res 1987;433:277-80.

25 Stanek B, Lischka A, Hostnagl H, Pollak A. Sympathoadrenal response to hypoglycaemia in infants. Eur 7 Pediatr 1988;148:253-6.

26 Anagnostakis DE, Cardinois R. Urinary catecholamine excretion and plasma NEFA concentration in small for excretion and plasma NEFA concentra
dates infants. Pediatrics 1971;47:1000-9.

27 Hughes D, Murphy JF, Dyas J, Robinson JA, Riad-Fahmy D, Hughes IA. Blood spot glucocorticoid concentrations in ill preterm infants. Arch Dis Child 1987;62:1014-8.

28 Anand KJS, Yacoub MY, Sippell WG, Aynsley-Green A. Endocrine control of glucose homeostatis during cardiopulmonary bypass (CPB) and cardiac surgery in full term neonates. $\mathcal{F}$ Endocrinol 1985;104(suppl): 140 . 